\title{
Factors that influence Translation and Interpreting technology adoption by university instructors, through the lens of the Technology Acceptance Model (TAM)
}

\author{
Seb Dianati ${ }^{1}$ *, Nantana Taptamat ${ }^{\mathbf{1}}$,Akiko Uchiyama ${ }^{\mathbf{1}}$, Natsuko Akagawa ${ }^{\mathbf{1}}$ \\ ${ }^{1}$ School of Languages and Cultures, University of Queensland, Brisbane, Australia
}

\begin{tabular}{|c|c|c|c|}
\hline Received: 27.02.2022 & Accepted: 05.03.2022 & Published: 05.03.2022 & Final Version: 10.03.2022 \\
\hline
\end{tabular}

\begin{abstract}
The purpose of this study was to use mixed methods to examine the factors that contribute to the adoption of translation and interpreting (T\&I) technologies by university instructors. The qualitative outcomes aimed to ascertain which technologies are currently being used in Australian universities, in the categories of web-search, CAT, mobile and tablet, and language lab software and hardware. An infographic was used to help display the technologies in these four domains. The findings of the quantitative analysis indicate that the frequent use of T\&I technologies in instructors' current practice significantly affected their intention to use technologies in the future. However, their experiences in both teaching and using such technologies were not factors that influenced their future use. The instructors who viewed T\&I technologies favourably tended to recommend these tools to their friends and family; they believed that these tools helped them improve the accuracy of their job performance and secure their current job, and generally gave them an advantage in the employment market. At the same time, the instructors in our study faced some issues in using T\&I technologies, such as the accuracy of their output and the lifespan of the software. Regardless of the challenges they faced, if an instructor perceived T\&I technologies to be useful, they tended to express an intention to continue to use such technologies in the future.
\end{abstract}

Keywords: translation and interpreting technology, technology acceptance model, Computer Assisted Tools, university technology

\section{Introduction}

Two distinct challenges have arisen in the Translation and Interpreting (T\&I) field due to COVID19. The first is the increased isolation of practitioners, many of whom have been forced to work remotely, often with a greater reliance on technology. Second, the pandemic has meant that instructors face constant pressure to keep up to date with various technologies, often in a 'sink or swim' situation. Consequently, there is a need to examine the factors that promote or hinder technology adoption within academia so that students and staff are better equipped with the technological skills for professional practice. There are only a handful of researchers whose work is dedicated to understanding the motivations for T\&I technology adoption by academics. Given the growing influence of globalisation and outsourcing in the T\&I industry, both students and teachers need to develop an understanding of the broad range of tools available to them. This paper aims to 
determine which technological tools are being used in current teaching practice in order to offer a snapshot of the extent of technology use in teaching and learning in Australia's higher education system. As research in this space is still in its infancy, the review of the literature provided here draws on international studies to demonstrate the importance of such research and support the growing need to understand the predictors of technology adoption by academics in T\&I education. Translators and interpreters are increasingly being asked to be better equipped with digital skills. Tomasello (2019, p. 3) notes that "the new competencies that will be asked from translators to thrive in the AI (Artificial Intelligence) era will not only regard operative, managerial, technical or digital knowledge, but also empathy, emotional intelligence and creativity". Tomasello (2019) sees this duality as something that will always distinguish T\&I practitioners from technology and believes that there will always be a need for the human translator and interpreter. Hence, while this research focuses on a greater understanding of the adoption of technologies in T\&I, it does so without discounting the continuing and significant role of human translators today and into the future.

While the adoption of technologies by professionals varies, current formal training in these technologies in higher education is still sporadic. A study by Kerremans et al. (2019) presents the first results of a survey on the technology use of 188 public service interpreters and translators in Australia and Europe. Their study examined how previous training in the use of technology tools was correlated with the current use of them in practice. The results show that European T\&I specialists used these tools more willingly, more frequently, and with greater diversity than their Australian counterparts (Kerremans et al., 2019, p. 118). This research is important for the present study as it reveals that only 10 to $18 \%$ of practitioners in Australia have been trained to use one or more of the existing technologies. As Kerremans et al. (2019, p. 119) note that "formal studies positively influence the frequency of use of CAT tools", the lower rate of widespread technology adoption in Australia may reflect the lack of opportunities for formal training. The lack of a framework of competency in Australia may also be contributing to the low adoption rates, as there is no overarching set of standards that relate to technology. The European Master's in Translation (EMT) may provide a guide for Australian academics in this regard, as technology represents one of the five themes of competency (EMT, 2021). Koponen's (2016, p. 143) literature review identified a shift towards the frequent use of Machine Translation (MT) post-editing in industry, research, and education. Our paper draws on the significance of this research to argue for the deeper understanding of the predictors of and barriers to technology adoption that academics face, which in turn will help to broaden the sector's understanding of the availability and use of technologies for T\&I. This is what Cheung and Vogel (2013) mean when they suggest that the benefits of technology must be accepted by academics before it will be accepted by students.

In response to the growing presence of Machine Translation (MT) in the industry, Mellinger (2017) proposes the incorporation of translation technologies in courses across the translation curriculum, rather than offering technology content as a 'bolt-on' fix or even as a standalone course. Kenny and Doherty (2014) state in their design of a Statistical Machine Translation (SMT) course that there is a need for academics to incorporate technological tools within their course design. Similarly, Koponen (2016, p. 10) has found that the MT and post-editing course at their university "appears to have succeeded in fostering a positive attitude, as most of the students commented on seeing MT in a more positive light after the course". One challenge encountered by Koponen that has relevance for teachers in Australia is that "the course is offered to students in all language and translation subjects, which makes it difficult to provide materials for all the language pairs they may be working in" (Koponen, 2016, p. 13). As most T\&I programs in Australian universities offer more than one language pair, coursework applications must be aligned with programme-level learning 
14 Dianati et al.: Factors that influence Translation and Interpreting technology adoption by university instructors, through the lens of the Technology Acceptance Model (TAM)

objectives. However, for any technology adoption to occur, understanding the factors that may hinder or facilitate its use in the higher education sector is becoming increasingly important.

According to Mellinger (2017), the necessary training includes, but is not limited to, terminology management, controlled authoring, post-editing, and engine tuning. While our study does not focus on these factors individually, it situates T\&I technological tools more broadly as a component of future T\&I curricula. Two decades ago, Kiraly (2000, p. 284) suggested that "student learning needs to be situated and contextualized within the larger act of translation, and professionally oriented tasks embedded in a series of courses or modules". This study argues that T\&I technologies are now firmly situated within professional practice and therefore need to be incorporated into formal instruction.

There is also a lack of research in T\&I digital training. The review by Christensen et al. (2017, p. 10) of 192 papers published in translation studies journals between 2006 and 2016 found that only 8 papers, or $2 \%$, were concerned with technology, and fewer than half of these related to training. Christensen et al. (2017, pp.18-19) note: "The translators of the future will need more training in IT, they will need training in new forms of translation, and they will need to develop their competencies as efficient communicators and text producers". Escobar-Rodríguez et al. (2014) observe that job performance is one of the strongest predictors of the adoption and use of technologies (see also Venkatesh et al., 2003). Hence, there is a need for researchers to evaluate the factors that affect technology adoption, which, in turn, can affect how T\&I courses are developed around technological tools. To do so, our research used the Technology Acceptance Model (TAM) as its underlying theoretical and conceptual framework, even though it was not used in our actual analysis due to sample size restrictions.

\section{Literature review}

\subsection{Conceptual Framework: Technology Acceptance Model (TAM)}

TAM is a model that helps to understand, explain, and predict an individual's acceptance of technology, and is one of the most well-known, cited, and supported models for predicting the adoption of any technology. It builds on earlier work with the Theory of Reasoned Action and the Theory of Planned Behaviour, which argue that the intent to use a technology will depend on the individual's attitudes towards that technology (Ajzen, 1987). Davis (1989) incorporated two key factors into his original model: Perceived Usefulness (PU) and Perceived Ease of Use (PEOU). These two constructs help predict usage intentions and adoption behaviours relating to the acceptance of technology. The revised model, the Extended Technology Acceptance Model (TAM2), aimed to include other factors such as social influences, voluntariness, subjective norms, image, job relevance, output quality, and results demonstrability to help explain intention to use (Venkatesh \& Davis, 2000). TAM2 explains up to $60 \%$ of the variance in the usefulness of a given tool (Venkatesh \& Davis, 2000). While TAM has been used in a variety of contexts and settings, including teaching and learning, there is limited research on TAM in the context of T\&I technologies (Yang \& Wang, 2019). PU and PEOU, together with facilitating conditions and social influences, were the four main constructs used in our research design. The TAM2 model was used as a framework for the study rather than in the analysis itself, as the sample size was not large enough to enable its use for construct validity and reliability. Hence, TAM2 guided our research to find good predictors in our regression model, even though it was not explicitly used in the analysis. 


\subsection{T\&I Technologies and TAM}

It is useful to examine other studies that have incorporated TAM in the context of T\&I technology adoption. For instance, there is research that indicates that perceived usefulness (PU) is a strong predictor of Machine Translation (MT) usage (Yang \& Wang, 2019). Furthermore, experience has been shown to influence PU, and PEOU to influence motivation (Yang \& Wang, 2019). Other research has indicated that PEOU, PU, and motivation are strong predictors of the intention to use Google Translate, and furthermore, that PU and motivation strongly correlate with PEOU (AlMaroof et al., 2020). Another study found that effort and performance expectancy strongly influenced the perceived usefulness and overall benefit of post-editing tools, indicating that "participants perceive the amount of time and work that is saved when post-editing to be a greater benefit than the overall utility of post-editing" (Yang et al. 2021, p. 101). Moreover, PU influenced attitudes towards Neural Machine Translation (NMT) adoption, and social influences also played an influential role in the extent of its adoption (Chun, 2020). Social influences tend to positively impact technology use and behaviour (Liao et al., 1999; Lucas \& Spitler, 2000). Habit is another indicator that needs to be mentioned, as once technologies are used more frequently, they are more likely to be used instinctually, and with little cognitive effort (Chávez Herting et al., 2020). Furthermore, if the use of technology becomes pleasurable, hedonism has been shown to positively affect participants' intention to use that technology (Van der Heijden, 2004).

Other factors that have been found using TAM to influence T\&I technology adoption include control, image, and subjective norms (Rossi \& Chevrot, 2019). There is also evidence to suggest that satisfaction (as enjoyment) and efficiency (as usefulness) strongly influence T\&I technology adoption. Furthermore, emotive responses and feelings towards technology have also been shown to influence intention to use (Koskinen \& Ruokonen, 2017). With regard to Computer Assisted Translation (CAT) tools, Effort Expectancy (EE), Performance Expectancy, Social Norms, Playfulness, and Self-Management were all considered strong influences on the continued adoption of CAT tools (Estellés, 2015). The next section outlines some of the strongest predictors of technology adoption that were used as independent and dependent variables in our study.

\section{Independent Variables}

\section{Perceived Ease of Use (PEOU)}

PEOU relates to the degree an individual believes using a particular technology will be free from effort. Ease of use relates to the level of mental effort it takes to perform a task and the extent to which it is understandable (Davis, 1993). For instance, Venkatesh and Davis (1996) observed that the specific determinants of an individual's PEOU relate to that individual's computer self-efficacy (Venkatesh \& Davis, 1996). PEOU and PU are considered the two strongest predictors of intention to use.

\section{Perceived Usefulness (PU)}

PU relates to the degree to which an individual believes using a particular technology is useful in their role. Usefulness can be understood in several ways. It can relate to whether the technology increases the quality of one's work through greater control or increased efficiency. Interestingly, PU was found to be $50 \%$ more influential than ease of use in determining technology usage (Davis, 1993). Underpinning usefulness are the individual's reaction to using the technology, their intention to use it, and their actual usage. However, PEOU and PU influence one another. Once technology 
16 Dianati et al.: Factors that influence Translation and Interpreting technology adoption by university instructors, through the lens of the Technology Acceptance Model (TAM)

becomes easier to use, the more useful that technology becomes, and vice versa (Venkatesh \& Davis, 2000). This is by far the strongest predictor of technology adoption that was found in a critical review of TAM (Legris et al., 2003).

\section{Facilitating Conditions (FC) and Attitudes Towards Use (ATU)}

One of the greatest weaknesses of the original TAM model is that it did not consider external variables. Facilitating conditions as a construct aims to identify the perceived enablers or barriers in an environment. Technical support, for example, is one of the most influential facilitating conditions affecting technology adoption in teaching and learning (Williams, 2002). It also influences attitudes to use (Ngai et al., 2007). This is why support related questions were incorporated in the survey design. Attitude is important when combined with other variables (Alharbi \& Drew, 2014). It is a strong predictor when technology adoption is voluntary (Chau, 2001). ATU evaluates whether the individual has a positive view of the technology.

\section{Dependent Variable: Behavioural Intention to Use}

Behavioural Intention (BI) refers to an individual's willingness to use technology. BI acts as the dependent variable, whereas the other factors listed are all independent. This study aims to evaluate the factors that affect individuals' intention to use T\&I tools.

Initially, the purpose of this research was to determine which constructs of the TAM were validated by instructors using translation and interpreting (T\&I) technologies, using Factor Analysis. However, as noted above, the survey sample size was not large enough to continue in this direction. Instead, correlation of the four main constructs - PU, PEOU, FC, and ATU - is used to examine which factors are correlated with BI and to what extent they are related. Lastly, the study aims to examine which factors are good predictors of instructors' willingness to use T\&I technologies. Understanding these predictors will facilitate the development of technology-embedded T\&I curricula in Australia. It will also help to provide more relevant support to academics to better align theory with current practice. The end objective is for T\&I researchers and educators to become more comfortable teaching technology in the classroom.

\section{Research Questions}

Based on the above objective, the research questions directing this study are:

RQ1: What T\&I technologies are currently being used by instructors in translation and interpreting courses?

RQ2: What factors affect the adoption of T\&I technologies by instructors in their courses?

\section{Research Design and Instrument}

The research involved an online survey, which included two sections relating to enabled systematic and relative data capture respectively. Section 1 involved fixed response items to elicit demographic information: affiliation, current appointment, levels of teaching (e.g., undergraduate or master's level), gender, years of teaching, previous experience using T\&I technologies, areas of teaching, and current use of technologies. Section 2 included questions developed from previous TAM studies using standard scales as a basis for designing survey questions. The TAM model comprised 32 items (see Table 5), which measured 'perceived usefulness' ( 7 items), 'perceived ease of use' (5 items), 'facilitation conditions' (5 items), 'social influences' (6 items), 'behavioural 
intention' ( 3 items), and 'attitude toward usage' ( 6 items). The scales for these items were either a five-point or a seven-point positively packed Linkert scale, where 7 or 5 denoted strongly agree. The questionnaires were constructed and administered using Microsoft Forms, allowing the researchers to collect and export the responses from participants using the appropriate software for data analysis.

\subsection{Participants}

The participants included twenty-one T\&I instructors from a broad spectrum of Australian universities, who teach translation and/or interpreting at university level. The online survey was administered via email using Microsoft Forms. Participation was voluntary and data were collected anonymously. Most participants were female $(n=14)$, with males making up the remainder $(n=7)$. Demographic data suggest that half of the participants taught master's degree students $(n=11)$, while others taught undergraduate courses $(n=5)$, and the remainder taught a combination of both. Most of the participants used technological tools in T\&I more than once a week ( $n=15)$, while 2 did not use them at all, and the remainder only used technology 'when needed'.

\subsection{Survey Data Analysis}

The survey used open-response scales, to provide a better understanding of the factors that influence participants' use of technology. Once the ethical clearance application was approved, the survey was sent out to potential participants. Once the survey closed, the responses were converted to numerical input for test statistics. Data screening was carried out to identify entry errors and determine whether the data met the assumptions for inferential statistical analysis. Preliminary descriptive analysis was conducted to examine some statistics such as central tendencies, variability, and normality. Although we initially planned to use TAM and a structural equation modelling to test our hypotheses, the survey did not receive enough responses for us to do so. In addition, the participants included only a small number of instructors specialising in T\&I technologies in Australia. To answer research question 2, we performed descriptive statistics and two non-parametric analyses: Kendall's Tau Correlation and ordinal regression to explore the relationships between factors influencing instructors' use of T\&I technologies. Statistical Package for the Social Sciences (SPSS) was used for the descriptive, correlation, and regression analysis.

\section{Findings and Discussion}

This section presents the findings of our qualitative and quantitative analyses relating to each research question.

\subsection{RQ1 Emerging trends in T\&I technologies in Australian Higher Education}

In response to RQ1, regarding the T\&I technologies that were currently being used by academics in translation and interpreting courses, Figure 1 outlines the responses in a thematic infographic. Responses were grouped into themes in terms of which technological area the product or service belonged to. The results were then triangulated with the literature to examine the technologies' usefulness and ease of use for future practitioners.

In terms of web-search software, Google Translate, YouDao, BaiDu, ELAN languages, and Microsoft Translator were identified as the most commonly used web-search software, while AntConc and Voyant were seen as useful Corpus-based tools. For instance, YouDao has been a strong competitor for Google Translate and Microsoft Translator because of its ability to detect and 
18 Dianati et al.: Factors that influence Translation and Interpreting technology adoption by university instructors, through the lens of the Technology Acceptance Model (TAM)

correct errors by non-Chinese language learners using NMT approaches (Fu et al., 2018). However, it seems that in the case of complex business contracts it is limited in its ability to differentiate between plural and singular words, and handle long sentences, passive/active tenses, and conditional forms (Zhang, 2019, p. 77). As Google is banned in China, it is important to note the influence of BaiDu as a search engine in that country. BaiDu appears to have an advantage over Google Translate in regard to coding time and cultural relevance, while Google is superior in terms of accuracy and clarity (Md Abdur et al., 2019). However, recent findings suggest that BaiDu has improved its usability and applications (Liu et al., 2021).

In relation to Computer-Assisted Translation (CAT) tools, participants in this study nominated WordFast, Trados, MateCat, and Memsource as the most useful, and saw WinCaps, Goreact, and Ageisub as useful subtitling CAT technologies. WordFast has been identified as the second most common and widely used CAT tool, after SDL Trados (Li, 2020). Despite this, WordFast has been shown to be the easiest technology to use for PowerPoint presentations, and to be more accurate than Memsource and Trados (Jie, 2021). However, with HTML and word functions, there is evidence to suggest that Trados is less complicated and less time intensive than WordFast (Al-Hammar, 2019). In more nuanced areas such as field-work documentation, especially around Indigenous language preservation, ELAN has become the benchmark for the annotation of verbal communications. When comparing Memsource and MateCat (as good CAT alternatives to non-desktop applications), it was found that MateCat was considerably more user-friendly than Memsource and that the industry lacks awareness of the alternative tools in circulation (Rodríguez Vázquez et al., 2018). In the subtitling category, Agiesub was considered useful for YouTube subtitling as it allows for the accurate inclusion of idiomatic expressions (Nugroho \& Basari, 2019), while WinCaps might be more useful for live intra-lingual subtitling (Robert \& Remael, 2017). With respect to MT, technologies should also aim to make the T\&I role more cost-effective and efficient, even if it is not always completely accurate (Bowker \& Ciro, 2015).

In terms of Learning Management System Integration, GoReact was considered less well integrated for language learning flipped classroom environments than its competitor CritiqueIt (Rumzan, 2020). The Televic suite of products was identified as optimal for interpreter training. This includes their Interpreter Q interpreter training system, which combines conference hardware and intuitive training software, alongside their language lab software AVIDAnet Live. Televic has become a leading player in T\&I training, evaluation, and certification, and the release of their Translation Q software across forty different institutions has produced positive outcomes in relation to authenticity, objectivity, and efficiency (Wylin \& Van Egdom, 2021).

For mobile and tablet usage, Livescribe was the preferred pencil annotation tool. Among digital translation pens, LiveScribe is a leader. For instance, it works through "Optical Character Recognition (OCR) and Text to Speech (TTS) conversion, using concatenation synthesis. OCR is achieved using image processing, character extraction, and classification" (Alzubaidi et al., 2021, p. 1). It has been shown to be easy to use and especially useful in real-time Arabic translation (Alzubaidi et al., 2021, p.1). Zoom subtitling and live transcription functions alongside Bidule equipment were also listed as important technological tools. While this list is not exhaustive, the infographic below aims to highlight the responses we received regarding these tools in the various categories and offers a snapshot of some of the hardware and software being used in teaching and learning in Australia. 


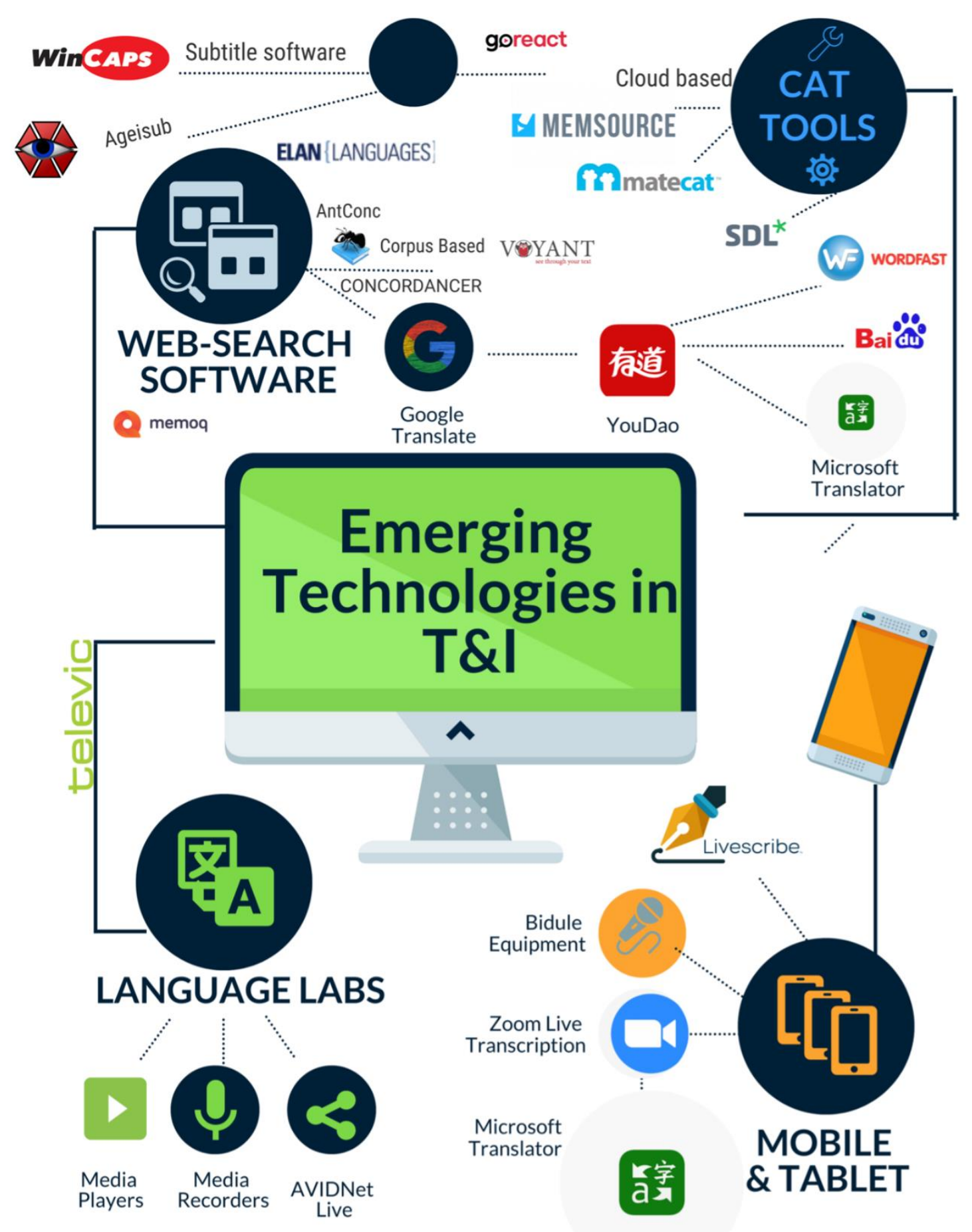

Figure 1. A list of various technological tools used by T\&I academics in Australia.

\subsection{RQ2 Factors that influence the adoption of $T \& I$ technologies by instructors in Australia}

Twenty-one instructors ( 7 male and 14 female) provided demographic information about their experience in teaching and using T\&I technologies, their work appointments, and the levels of courses they taught. While 11 had continuing appointments, 10 were casual instructors. Additionally, 11 instructors taught master's degree courses, 1 taught a diploma course, 5 taught bachelor's courses, and 4 taught both undergraduate and postgraduate courses. Most instructors were veteran teachers: 7 had 6-9 years' teaching experience and 11 had more than 10 years' experience. Only 2 of the 
20 Dianati et al.: Factors that influence Translation and Interpreting technology adoption by university instructors, through the lens of the Technology Acceptance Model (TAM)

respondents had been teaching for less than two years, and only 1 had been teaching for 2-5 years. The instructors rated their previous experience using T\&I technologies on a scale from 1 (no experience) to 7 (the highest level of experience). Their self-rated scores were fairly evenly distributed across the different levels. Figure 2 summarizes the instructors' intention to use T\&I technologies in the future in terms of their teaching experience and their previous experience in using such technologies.

Instructors' opinions on whether they intended to use T\&I technologies by their teaching experience (years)

\begin{tabular}{|l|r|r|r|r|r|}
\hline $\begin{array}{l}\text { Years of } \\
\text { teaching }\end{array}$ & Strongly disagree & Disagree & $\begin{array}{r}\text { Neither agree nor } \\
\text { disagree }\end{array}$ & Agree & Strongly agree \\
\hline$<2$ years & & & 01 & & 1 \\
\hline $2-5$ years & & & & & \\
\hline $6-9$ years & & & 01 & & 000006 \\
\hline 10 year+ & & 01 & 01 & & 003 \\
\hline
\end{tabular}

Instructors' opinions on whether they intended to use T\&I technologies by their previous experience of using T\&I technologies (level 1-7)

\begin{tabular}{|c|c|c|c|c|c|}
\hline $\begin{array}{l}\text { Previous } \\
\text { experience }\end{array}$ & Strongly disagree & Disagree & $\begin{array}{r}\text { Neither agree nor } \\
\text { disagree }\end{array}$ & Agree & Strongly agree \\
\hline 1 & & & & 1 & 1 \\
\hline 2 & & & 1 & & 1 \\
\hline 3 & & & 1 & & $\mathrm{O} 2$ \\
\hline 4 & & 1 & & $\bigcirc 2$ & \\
\hline 5 & & & 1 & 1 & 1 \\
\hline 6 & & & & & $\mathrm{OO}_{3}$ \\
\hline 7 & & & & & 0003 \\
\hline
\end{tabular}

Figure 2. Instructors' opinions on their intention to use T\&I technologies in the future, based on their teaching experience and levels of experience using the technologies.

According to Figure 2, most of the instructors strongly agreed that they would use T\&I technologies in the future, regardless of their past experience with teaching and using such technologies. To determine the factors influencing participants' adoption of translation and interpreting technologies, we examined the relationships between 20 factors and instructors' indications of how likely they were to use T\& I technologies in the future. The descriptive analysis suggested that the participating instructors tended to value technologies highly, in terms of their usefulness, facilitation conditions, and behavioural intention. More than $80 \%$ of participants thought technologies had "some" to "extensive" benefits for their current and future job attainment, and in enabling students to carry out activities. Similarly, more than $60 \%$ believed technologies to be "somewhat" to "completely" useful for accuracy of performance and teaching and learning. We also conducted correlation and regression analysis to further investigate the factors influencing instructors' intention to use technologies. Their opinions on some aspects of using T\&I technologies are summarized in Figure 3. 


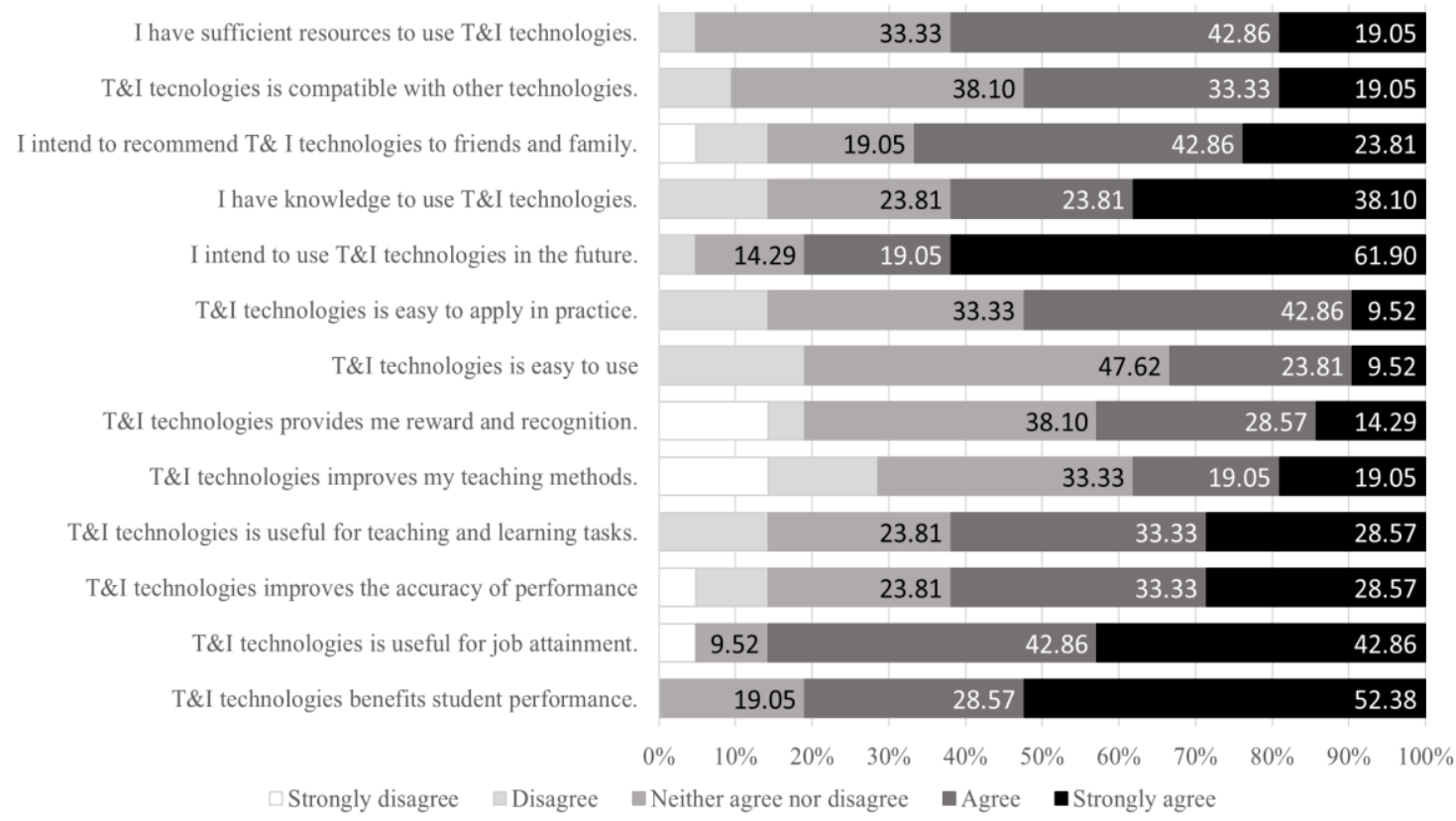

Figure 3. Instructors' views on using “T\&I technologies".

As shown in Figure 3, 52.38\% of the participants strongly agreed that T\&I technologies improved students' performance, and $42.86 \%$ strongly agreed that they were useful for current and future job attainment. Furthermore, $33.33 \%$ agreed or strongly agreed that T\&I technologies were useful for teaching and learning, and $28.57 \%$ either agreed or strongly agreed that they improved the accuracy of performance. In contrast, $9.52 \%$ strongly agreed that T\&I technologies were easy to use and apply in practice. Around $70 \%$ of the instructors had a firm belief (strongly agreed) that they would use T\&I technologies in the future, while $70 \%$ thought that they would recommend such technologies to their friends and family (43\% agreed, $24 \%$ strongly agreed). This is similar to the perceived influence of friends and family in other industries (Nidhi et al., 2020). However, Ajibade (2018) provides a counter-narrative that the influence of friends and family is mainly evident when it comes to the personal use of technology rather than organisational use.

To examine the factors that might affect the use of T\&I technologies in participants' classrooms, we conducted correlation analysis and regression analysis. Because the study had a small sample size and some variables did not meet the assumptions of a parametric test, non-parametric analyses were performed. Kendall's Tau Correlation was used to examine the relationships between variables associated with the use of T\&I technology by the instructors. It was found that instructors' experience in teaching was negatively related to their experience in using T\&I technologies. In other words, experienced instructors used T\&I technologies less frequently than less experienced instructors. However, the relationship between years of teaching and years of using T\&I technologies was small and not statistically significant. Table 1 shows the correlation between instructors' intention to use T\&I technologies in the future and their demographic information.

Table 1. Kendall's Tau B Correlations of behavioural intention to use T\&I technologies in the future in relation to instructors' demographic information.

\begin{tabular}{|l|l|l|c|}
\hline Variables & $\begin{array}{c}\text { Intention } \\
\text { use T\&I }\end{array}$ & $\begin{array}{c}\text { Years of } \\
\text { teaching }\end{array}$ & $\begin{array}{c}\text { Years } \\
\text { using T\&I }\end{array}$ \\
\hline
\end{tabular}


22 Dianati et al.: Factors that influence Translation and Interpreting technology adoption by university instructors, through the lens of the Technology Acceptance Model (TAM)

\begin{tabular}{|c|l|l|l|l|}
\hline \multirow{2}{*}{$\begin{array}{c}\text { Years } \\
\text { teaching }\end{array}$} & Kendall's Tau B & -.02 & & \\
\hline \multirow{2}{*}{$\begin{array}{c}\text { Years of using } \\
\text { T\&I }\end{array}$} & $p$ value & .91 & & \\
\hline \multirow{2}{*}{$\begin{array}{c}\text { Frequency of } \\
\text { using T\&I I }\end{array}$} & $p$ value & .30 & -.22 & \\
\cline { 2 - 5 } & Kendall's Tau B & .12 & .25 & \\
\cline { 2 - 5 } & $p$ value & .04 & -.16 & -.30 \\
\hline
\end{tabular}

It came as a surprise to us that even the frequency of using T\&I technologies was negatively and moderately related to instructors' experience in using such technologies, although the relationship was not significant. It is worth noting that only the relationship between instructors' frequency of using T\&I technologies and their intention to use such technologies in the future was statistically significant. In addition, Kendall's Tau Correlation was used to examine the relationships between the factors associated with the instructors' intention to use T\&I technologies in the future. Of the 20 variables considered, 11 had statistically significant relationships with the instructors' intention to use T\&I technologies, as shown in Table 2.

Table 2. Kendall's Tau B Correlations of behavioural intention to use T\&I technologies in the future in relation to some variables.

\begin{tabular}{|l|l|l|l|}
\hline Variables & $r$ & $p$ & $\begin{array}{l}\text { Effect } \\
\text { size }\end{array}$ \\
\hline $\begin{array}{l}\text { I intend to recommend T\&I technologies to friends and } \\
\text { family. }\end{array}$ & $.72^{* * *}$ & $<.001$ & large \\
\hline $\begin{array}{l}\text { Using T\&I technologies are useful in current and future job } \\
\text { attainment. }\end{array}$ & $.65^{* *}$ & $<.001$ & large \\
\hline I have the resources available to me to use T\&I technologies. & $.56^{* *}$ & 0.005 & medium \\
\hline $\begin{array}{l}\text { Using T\&I technologies improve the accuracy of } \\
\text { performance. }\end{array}$ & $.54^{* *}$ & $<.001$ & medium \\
\hline $\begin{array}{l}\text { Using T\&I technologies motivate me and provides reward } \\
\text { and recognition. }\end{array}$ & $.51^{* *}$ & 0.006 & medium \\
\hline $\begin{array}{l}\text { I receive enjoyment or satisfaction when using T\&I } \\
\text { technologies. }\end{array}$ & $.47^{* *}$ & 0.013 & medium \\
\hline $\begin{array}{l}\text { Using T\&I technologies is useful to carry out my teaching and } \\
\text { learning tasks. }\end{array}$ & $.46^{*}$ & 0.007 & medium \\
\hline $\begin{array}{l}\text { The perceived benefits of T\&I technologies outweigh the } \\
\text { monetary cost. }\end{array}$ & $.45^{*}$ & 0.01 & medium \\
\hline $\begin{array}{l}\text { Using T\&I technologies benefits students in performing } \\
\text { activities. }\end{array}$ & $.44^{*}$ & 0.021 & medium \\
\hline $\begin{array}{l}\text { I have the knowledge needed to use T\&I technologies } \\
\text { effectively. }\end{array}$ & $.43^{*}$ & 0.042 & medium \\
\hline
\end{tabular}

As shown in Table 2, the intention to use T\&I technologies in the future was most significantly correlated to intention to recommend such tools to friends and family (with a large effect size, based on Plonsky and Oswald (2014)), compared to other factors. Other factors that have significant 
impacts on instructors' intention to use technologies in the future were perceived usefulness for job attainment ( $r=.68$; large effect size) and a belief in the availability of resources $(r=.56)$. Additionally, factors such as perceived usefulness for the accuracy of performance, for recognition, and for teaching and learning were moderately related to intention to use T\&I technologies in the future. Other significant medium influences were perceived usefulness for students' performance, personal enjoyment, and having sufficient knowledge to use the T\&I technologies, as well as the perceived benefits outweighing the cost.

Kendall's Tau B Correlations suggest that instructors' intention to use T\&I technologies in the future is not statistically correlated to their experience in either teaching or using T\&I technologies. In other words, their intention to use such technologies was not related to how long they had been either teaching or using T\&I technologies in the classroom. However, there was a moderate and significant relationship between their intention to use and their frequency of using T\&I technologies. In other words, the instructors who used T\&I technologies more often were more likely to use these technologies in the future. This is an extension of the finding of a study by Kerremans et al. (2019). While Kerremans et al. found that instructors who had received training in the use of particular tools were more likely to use those tools in their current practice, our study further suggests that those currently using the tools also planned to use them in the future. We also found that the instructors who thought that the organizational and technical infrastructure (such as resources and support) existed to support their use tended to believe that technologies were easy to use, which resonated with the findings of Ngai et al. (2007). On the other hand, users' perceptions of ease of use, usefulness, and facilitation conditions were not necessarily correlated with one another. In other words, when participants thought that T\&I technologies were useful, they tended to have a positive attitude towards using them.

Kendall's Tau B Correlation analysis also indicates that the instructors who were more inclined to use T\& I technologies would also recommend such technologies to their friends and family. The correlation between these two variables was strong. The proportion of instructors who thought that T\&I technologies were useful in the attainment of their current and future jobs ( $r=.65$; large) was statistically significant and relatively strong. This finding resonates with those of Venkatesh and Davis (2003) and Legris et al. (2003). In addition, those who indicated that they had adequate resources $(r=.56$; medium) expressed their intention to use T\&I technologies into the future. However, although they thought these technologies were useful, they did not necessarily think they were easy to use. Additionally, the perceived usefulness of the technologies appeared not to be influenced by their perception of whether or not their university provided sufficient infrastructure, resources, and technical support. Attitude toward usage was more strongly related to perceived usefulness than to perceived ease of use and facilitation.

To examine which factors are better predictors of an instructor's intention to use T\&I technologies, ordinal regression was carried out. The Proportional Odds model was used to predict instructors' intention to use T\&I technologies. The regression model included 3 factors, which involved the instructors' perceptions on the usefulness of T\&I technologies for reward and recognition, the usefulness of T\&I technologies for teaching and learning tasks, and their belief that they had sufficient resources and support. The test of parallel lines, $\chi^{2}(6)=6.87, p=.33$, indicated that the proportion across different thresholds was consistent; hence, the assumption of proportional odds was met. The analysis showed that the model containing three predictors presented a significant improvement in fit relative to the null model, LR $\chi^{2}(6)=14.06, p=.003$. Both the Pearson Chisquare $\left[\chi^{2}(6)=27.02, p=.92\right]$ and Deviance Chi-square $\left[\chi^{2}(6)=22.85, p=.98\right]$ tests were not significant, indicating a well-fitting model. The pseudo- $R$-square suggested that the model exhibits 
24 Dianati et al.: Factors that influence Translation and Interpreting technology adoption by university instructors, through the lens of the Technology Acceptance Model (TAM)

$32.3 \%$ fit improvement compared to the null model. McFadden's pseudo- $R$-square fell within .2 and .4 , indicating a strong model-fit improvement.

According to the regression analysis, an instructor's motivations in relation to reward and recognition, having sufficient resources, and perceived usefulness for the tasks were significant predictors of intention to use in the future. In addition, the overall parameter estimates suggested that, if instructors thought T\&L technologies was useful for reward and recognition, they expressed an intention to use such technologies; if they thought they had sufficient resources and support, they stated their intention to use T\&L technologies in the future; and if they perceived T\&L technologies to be useful for teaching and learning tasks, they expressed an intention to use such technologies. However, each individual predictor in the model was not significant, indicating that they are weak predictors.

\subsection{Challenges Faced by Participants}

Participants identified various challenges in using T\&I technologies, one of which was software reliability and technological accuracy. Some participants indicated that there was little interoperability between Machine Learning and Artificial Intelligence, while others commented that the technologies became outdated too quickly. One noted that the "terminology management is not good/intelligent enough". Mellinger (2017) agrees, observing that terminology management is not particularly effective. Another participant mentioned "malware in the software". While Gibert et al. (2020) believe that technology itself is an effective method of detecting malware, others such as Ragni and Vieira (2021) believe that most advances have been in the technology itself rather than its usability. To quote Ragni and Vieira (2021, p.1), "the majority of NMT research involving human stakeholders is directed towards advancing the state of MT development rather than ensuring the usefulness of NMT as a tool for professionals". Other challenges included the technologies being time-consuming to learn and quickly outdated, while others highlighted the lack of pedagogical benefits of the technologies, such as ease of use. For instance, one participant observed that if students were not competent in using technologies they struggled and could waste class time. Furthermore, if the technology was not working properly, it could interrupt the flow of the class and was seen as a disruption. Teaching online and internet connectivity was also raised as a concern. This was identified as an issue by Krutka et al. (2019), who suggested that connection problems in rural areas and intercultural experiences have an impact on student learning. Another major concern was the learning curve. One participant was unaware of the existence of technological tools, while another mentioned that there was a steep learning curve, although they found that using the technologies did become easier over time. Another participant was more concerned about the presumed technical proficiency required to learn new technologies. Lastly, there was the issue of resources. Two participants expressed concerns about a lack of skilled trainers and support personnel to teach staff and students how to use the available resources and observed that not all students had the same access to resources or equipment.

To explore whether the participants who were interested in adopting T\&I technologies in the future faced different challenges in using such technologies from those who did not intend to adopt them, cross tabulation of their responses to two questions on intentions for future use and the challenges of using T\&I technologies was conducted. Table 3 summarises instructors' views on their intention to use and the challenges of using T\&I technologies. 
Table 3. Instructors' views on their intention to use and the challenges of using T\&I technologies

\begin{tabular}{|c|c|c|c|}
\hline $\begin{array}{l}\text { Somewhat } \\
\text { unlikely }\end{array}$ & $\begin{array}{l}\text { Neither likely } \\
\text { nor unlikely }\end{array}$ & Somewhat likely & Very likely \\
\hline $\begin{array}{l}\text { - Accuracy and } \\
\text { very time- } \\
\text { consuming for } \\
\text { proofreading (1) }\end{array}$ & - Cost (1) & $\begin{array}{l}\text { - Accuracy (1) } \\
\text { - Reliability (1) } \\
\text { - Software design (1) }\end{array}$ & $\begin{array}{l}\text { - Malware in the software (1) } \\
\text { - Cost (2) } \\
\text { - Ease of use (2) } \\
\text { - Learning curve (2) } \\
\text { - Expiry date (1) } \\
\text { - Technical issues during class } \\
(1) \\
\text { - Technical support (2) } \\
\text { - Unaware of tools existence } \\
\text { (1) } \\
\text { - Accuracy (1) } \\
\text { - Integration with ML and/or } \\
\text { AI (1) } \\
\text { - Students aptitude or } \\
\text { confidence (1) }\end{array}$ \\
\hline
\end{tabular}

One instructor who stated that they were somewhat unlikely to use T\&I technologies in the future observed that "It does not translate accurately. Proofreading can be very time-consuming". Such perceived issues relating to accuracy and cost were found across the categories associated with instructors' intention to use technologies. One instructor who stated that they were highly likely to use T\&I technologies in the future commented that "Translation technology may have a steep learning curve at the beginning; however, once you are accustomed to using technology, it gets easier to get proficient with new tools". When the instructors' perceived challenges were viewed as (somehow) manageable, instructors were more likely to express an intention to use T\&I technologies in the future. However, this matter requires further investigation.

\subsection{Implications}

The implications of our findings for pedagogical practice focus on university instructors who teach translation and/or interpreting. This section first discusses the technologies that are currently used and their application in the classroom. The discussion then further considers the implications for administrators and policymakers, particularly in relation to providing adequate resources and support for teaching staff to enhance their perceptions of and proficiency in using translation and interpreting technologies.

First, we surveyed their current use and classified the emerging technologies in T\&I into 4 groups - mobile and tablet, language labs, web-search software, and CAT tools. An understanding of which tools are currently being used by educators could provide the basis for an introductory unit on T\&I tools for university teaching and learning. The current study responds to Schjoldager et al.'s (2017) observation that there is a need to provide T\&I training in these technologies. However, without understanding which tools are being used, and how, it is likely to be difficult for academics to adopt technology-embedded teaching practices. This is where this study can make a small but significant contribution to the field by offering an environmental scan of the current technologies used by instructors as a springboard for further adoption of and training in these tools for students in the future.

Second, it was found that instructors' attitude toward usage is statistically related to perceived usefulness, perceived ease of use, and facilitation. In other words, when users feel that translation 
26 Dianati et al.: Factors that influence Translation and Interpreting technology adoption by university instructors, through the lens of the Technology Acceptance Model (TAM)

and interpreting technologies are useful, easy to use, and they have the resources to support their use, they tend to have a positive attitude towards their use. Kerremans et al.'s (2019) finding that only 10 to $18 \%$ of Australian T\&I practitioners had learned how to use T\&I technologies may also be reflected in the lack of use of such tools in Australian higher education. It is critically important for the Australian higher education sector to promote the use of such tools and offer a competitive edge in their translator and interpreter training. In response to this need, Australian universities could: embed the use of such tools across their T\&I curriculum (Mellinger, 2017); encourage academics to use these tools in their classes (Kenny \& Dehert, 2017); and provide software training for academics (Kerremans et al., 2019; Schjoldager et al., 2017).

In addition, some of our survey participants identified challenges relating to software problems, cost, the steep learning curve, class activity issues, scarcity of resources, and difficulty of use. Software issues, for example, included malware, software design, and the reliability and accuracy of output. However, these issues are consistent across all technologies, not just those in the T\&I field. Some participants noted that technologies become outdated quickly and it is expensive to keep up to date. As Gefen, Karahanna and Straub (2003) found that trust is an important indicator of technology adoption, this may be a contributing factor to a lack of trust in the technologies in the current study. Additionally, if academics perceive T\&I technologies to be highly useful, they tend to use these technologies even though they may face some challenges in doing so (Bowker \& Ciro, 2015). It is important for institutions to help academics address these issues. Australian universities should provide appropriate and adequate support and resources to promote academics' positive perceptions of the usefulness and ease of use of T\&I technologies and work to facilitate increased technology adoption.

\subsection{Limitations}

Four limitations of this study are recognised. The first of these relates to the small sample size, due in part to the relatively small number of teachers in this space in Australia. The second limitation is that the research was conducted only within Australia; hence, caution should be exercised in applying these results, especially where political, cultural, and social factors surrounding technology use need to be considered. Third, the ranking scales used in the study were not uniform; in future iterations, a consistent Likert scale should be considered, rather than 1-5 and 1-7 scales being used interchangeably. Lastly, greater care could have been taken in the research design to make the distinction between translation and interpreting technologies more explicit.

\subsection{Recommendations}

Research into the factors influencing academics' adoption of technology in translation and interpreting within Australia is a relatively new area, so there is considerable space for future research to confirm, extend, or question our findings. The conclusions arrived at by this study provide some clear directions for future investigations based on diverse groups of participants such as students and instructors in different countries. To enable more robust generalization procedures, such as using structural equation modelling, our experience suggests a need to recruit a sufficiently large sample size for future studies. Future research could examine a larger cross-cultural sample with a larger sample size to evaluate whether there are differences in different country settings. Investigations could also be extended to other groups of participants, such as students in T\&I programs and $\mathrm{T} \& \mathrm{I}$ practitioners. 


\section{Acknowledgements.}

We would like to acknowledge the School of Languages and Cultures Strategic Research Fund Initiative at the University of Queensland who provided financial support totaling \$15,562.40. A.U., N.A. S.D. first proposed the idea for the project, S.D, A.U. and N.A. operationalised the project, N.T. processed the results, and N.A., A.U. and N.T. provided insights into the analysis. S.D. was the lead author of the paper.

\section{References}

[1] Ajibade, P. (2018). Technology Acceptance Model limitations and criticisms: Exploring the practical applications and use in technology-related studies, mixed-method, and qualitative researches. Library Philosophy and Practice.

[2] Alharbi, S., \& Drew, S. (2014). Using the Technology Acceptance Model in understanding academics' behavioural intention to use learning management systems. International Journal of Advanced Computer Science and Applications 5 (1). https://doi:10.14569/ijacsa.2014.050120

[3] Ayyagari, R. (2006). Examination of Hedonism in TAM research. Information Processing \& Management 42 (1), 325-326. https://doi:10.1016/j.ipm.2005.03.001

[4] Bowker, L., \& Buitrago, C. (2015). Investigating the usefulness of machine translation for newcomers at the public library. Translation and Interpreting Studies 10 (2): 165-186. 10.1075/tis.10.2.01bow

[5] Castilho, S. (2017). Is neural machine translation the new state of the art?. The Prague Bulletin of Mathematical Linguistics 108 (1), 109-120. https://doi:10.1515/pralin-2017-0013

[6] Chau, P. (2001). Influence of computer attitude and self-efficacy on IT usage behavior. Journal of Organizational and End User Computing 13 (1), 26-33.10.4018/joeuc.2001010103

[7] Cheung, R., \& Vogel, D. (2013). Predicting user acceptance of collaborative technologies: An extension of the technology acceptance model for e-learning. Computers \& Education 63 (1), 160-175. 10.1016/j.compedu.2012.12.003

[8] Christensen, T., Marian, F., \& Anne, S. (2017). Mapping translation technology research in translation studies. An introduction to the thematic section. Journal of Language and Communication in Business 56, 7-20. 10.7146/hjlcb.v0i56.97199

[9] Escobar-Rodríguez, T., Carvajal-Trujillo, E., \& Monge-Lozano, P. (2014). Factors that influence the perceived advantages and relevance of Facebook as a learning tool: An extension of the UTAUT. Australasian Journal of Educational Technology 30 (2), 1-19. 10.14742/ajet.585

[10]European Masters of Translation EMT. (2017). 'European Master's in Translation EMT competence framework accessed https://ec.europa.eu/info/sites/default/files/emt_competence_fwk_2017_en_web.pdf

[11] Field, A. (2021). Discovering Statistics Using SPSS: Introducing Statistical Method. 4th ed. CA: Sage.

[12] Gefen, D., Karahanna, E, and Straub, D. (2003). "Trust and TAM in online shopping: An integrated model". MIS Quarterly 27 (1), 51. 10.2307/30036519

[13] Gibert, D., Mateu, C., \& Planes, J. (2020). The rise of machine learning for detection and classification of malware: Research developments, trends, and challenges. Journal of Network and Computer Applications 153, 102526. 10.1016/j.jnca.2019.102526

[14] Kember, D., \& Ginns, P. (2021). Evaluating teaching and learning: a practical handbook for colleges, universities, and the scholarship of teaching. 4th ed. Routledge.

[15] Kenny, D. (2017). Human issues in translation technology. 1st ed. New York: Routledge.

[16] Koehn, P. \& Knowles, R. (2017). Six challenges for neural machine translation. Computation and Language (cs.CL) 1 (1).

[17] Koponen, M. (2016). Is machine translation post-editing worth the effort? A survey of research into postediting and effort. The Journal of Specialised Translation, 25, 131-148. 10.1007/s10590-014-9156-x

[18] Krutka, D., et al. (2019). Wise practices and intercultural understandings: A Framework for educator videoconferencing. Journal of Research on Technology in Education 51 (4), 356-376. $10.1080 / 15391523.2019 .1652869$

[19]Liao, S. et al. (1999). The adoption of virtual banking: an empirical study. International Journal of Information Management 19 (1), 63-74. 10.1016/s0268-4012(98)00047-4

[20] Lucas, H., \& Spitler, V. (2000). Implementation in a world of workstations and networks. Information \& Management 38 (2). 10.1016/s0378-7206(00)00059-8 
28 Dianati et al.: Factors that influence Translation and Interpreting technology adoption by university instructors, through

the lens of the Technology Acceptance Model (TAM)

[21] Yu, T., Yi Y., Lin, L., Mingming, S., \& Ping L. (2021). Multi-modal dictionary BERT for cross-modal video search in Baidu advertising. In Proceedings of the 30th ACM International Conference on Information \& Knowledge Management, 4341-4351.

[22] Ngai, E.W.T., Poon, J.K.L. \& Chan, Y.H.C. (2007). Empirical examination of the adoption of WebCT using TAM. Computers \& Education 48 (2): 250-267. 10.1016/j.compedu.2004.11.007

[23] Plonsky, L., \& Oswald, F.L. (2014). How big is "Big"? Interpreting effect sizes in L2 research. Language Learning 64 (4), 878-912. 10.1111/lang.12079

[24] Ragni, V., \& Lucas N.V. (2021). What has changed with neural machine translation? A critical review of human factors. Perspectives: 1-22. 10.1080/0907676x.2021.1889005

[25] Ruokonen, M., \& Koskinen, K. (2017). Dancing with technology: translators' narratives on the dance of human and machinic agency in translation work. The Translator 23 (3), 310323. 10.1080/13556509.2017.1301846

[26] Sharan, M., \& Tisdell, E. (2015). Qualitative research: A guide to design and implementation. 4th ed. New York: Jossey-Bass Wiley.

[27] Singh, N., Neena, S., \& Francisco J.L. (2020). Determining factors in the adoption and recommendation of mobile wallet services in India: Analysis of the effect of innovativeness, stress to use and social influence. International Journal of Information Management 50, 191-205. 10.1016/j.ijinfomgt.2019.05.022

[28] Tomasello, L. (2019). Neural Machine Translation and Artificial Intelligence: What Is Left for the Human Translator? 10.1080/1750399x.2018.1501639

[29] Teo, T. (2009). Is there an attitude problem? Reconsidering the role of attitude in the TAM. British Journal of Educational Technology 40 (6), 1139-1141. 10.1111/j.1467-8535.2008.00913.x

[30] van der Heijden, H. (2004). User acceptance of Hedonic information systems. MIS Quarterly 28 (4), 695.

[31] Venkatesh, V., Thong, J., \& Xu, X. (2012). Consumer acceptance and use of information technology: Extending the Unified Theory of Acceptance and use of technology. MIS Quarterly 36 (1), 157. $10.2307 / 41410412$

[32] Williams, P. (2002). The learning web: the development, implementation, and evaluation of internet-based undergraduate materials for the teaching of key skills. Active learning in higher education 3(1), 40-53. $10.1177 / 1469787402003001004$

[33] Wylin, B., \& Gys-Walt V. (2020). Big wrong data: Insights from developing and using an automatic translation revision tool with error memories. In Collated Papers for the ALTE 7th International Conference, Madrid, 130.

[34]Zhang, P. (2019). On translation of business contract by Youdao, 2019 International Conference on Modern Education and Economic Management (ICMEEM 2019), Xiamen University. 